\title{
When do the Recession Cones of a Polyhedral Complex Form a Fan?
}

\author{
José Ignacio Burgos Gil · Martín Sombra
}

Received: 3 September 2010 / Revised: 8 December 2010 / Accepted: 12 December 2010 /

Published online: 1 February 2011

(C) Springer Science+Business Media, LLC 2011

\begin{abstract}
We study the problem of when the collection of the recession cones of a polyhedral complex also forms a complex. We exhibit an example showing that this is no always the case. We also show that if the support of the given polyhedral complex satisfies a Minkowski-Weyl-type condition, then the answer is positive. As a consequence, we obtain a classification theorem for proper toric schemes over a discrete valuation ring in terms of complete strongly convex rational polyhedral complexes.
\end{abstract}

Keywords Polyhedral complex · Fan · Toric scheme · Tropical variety

\section{Introduction}

Toric schemes over a discrete valuation ring (DVR) were introduced and classified in [3]. Let $N \simeq \mathbb{Z}^{n}$ be a lattice on rank $n$ and set $N_{\mathbb{R}}=N \otimes \mathbb{R}$ for the associated real vector space. In loc. cit., toric schemes over a DVR are described and classified in terms of rational fans in $N_{\mathbb{R}} \times \mathbb{R}_{\geq 0}$. The toric scheme associated to a fan is proper if and only the support of the fan is the whole of $N_{\mathbb{R}} \times \mathbb{R}_{\geq 0}$. In this case, we say

Burgos Gil and Sombra were partially supported by the MICINN research project MTM2009-14163-C02-01. Burgos Gil was also partially supported by the CSIC research project 2009501001.

\section{J.I. Burgos Gil (凶)}

Instituto de Ciencias Matemáticas (CSIC-UAM-UCM-UC3M), Calle Nicolás Cabrera 13-15,

Cantoblanco, 28049 Madrid, Spain

e-mail: burgos@icmat.es

M. Sombra

ICREA \& Departament d'Àlgebra i Geometria, Universitat de Barcelona, Gran Via 585, 08007

Barcelona, Spain

e-mail: sombra@ub.edu

url: http://atlas.mat.ub.es/personals/sombra 
that the fan is complete. In the literature, such toric schemes are also called toric degenerations.

If we intersect a rational fan in $N_{\mathbb{R}} \times \mathbb{R}_{\geq 0}$ with the hyperplane $N_{\mathbb{R}} \times\{1\}$, we obtain a strongly convex rational (SCR) polyhedral complex in $N_{\mathbb{R}}$. Thus, given a SCR polyhedral complex in $N_{\mathbb{R}}$, it is natural to ask if it defines a rational fan in $N_{\mathbb{R}} \times \mathbb{R}_{\geq 0}$ and hence a toric scheme. One can also ask up to which extent these complexes classify toric schemes over a DVR.

In [5, Lemma 3.2], Nishinou and Siebert claim that any complete SCR polyhedral complex in $N_{\mathbb{R}}$ gives rise to a rational fan in $N_{\mathbb{R}} \times \mathbb{R}_{\geq 0}$, but their proof is incomplete because they do not check that the intersection of any two of the obtained cones is a common face. Moreover, in recent references, it is claimed without proof that any SCR polyhedral complex (without further hypothesis) defines a rational fan; see, for instance, $[7, \S 2.3]$.

This question is less innocent than it appears. Indeed, in Example 3.1 we exhibit an SCR polyhedral complex that does not define a rational fan. Hence it is not possible to associate a toric scheme to an arbitrary SCR polyhedral complex.

The main result of this note (Theorem 3.4) is that, if we assume that the support of a complex is connected and satisfies the Minkowski-Weyl condition (see Definition 3.3 below), then its recession cones do form a complex. In particular, this is true in the complete case, thus filling the gap in the proof of the Nishinou-Siebert statement. As a consequence, we show that proper toric schemes over a DVR of relative dimension $n$ are classified by complete SCR polyhedral complexes in $N_{\mathbb{R}}$ (Theorem 4.3).

The connectedness and the Minkowski-Weyl hypothesis are sufficient but not necessary. A wide class of examples whose recession cones form a complex but that may not satisfy the above hypothesis is that of extendable complexes (Definition 3.13). A consequence of our result is that any extendable SCR polyhedral complex defines a toric scheme.

The question of when an SCR polyhedral complex defines a fan is also relevant in tropical geometry: the definition of a tropical compactification of a subvariety of the torus depends on the construction of a toric scheme from an SCR polyhedral complex supported on the associated tropical variety. Since a tropical variety has a natural structure of SCR polyhedral complex that is extendable, our theorem implies the existence of such a toric scheme.

\section{Preliminaries on Polyhedral Complexes}

Let $N \simeq \mathbb{Z}^{n}$ be a lattice on rank $n$. We write $N_{\mathbb{R}}=N \otimes \mathbb{R}$ for the associated real vector space and let $M_{\mathbb{R}}=N_{\mathbb{R}}^{\vee}$ be its dual space. The pairing between $x \in M_{\mathbb{R}}$ and $u \in N_{\mathbb{R}}$ will be denoted by $\langle x, u\rangle$. A polyhedron of $N_{\mathbb{R}}$ is a convex set defined as the intersection of a finite number of closed halfspaces. It is strongly convex if it contains no line. It is rational if the closed halfspaces can be chosen to be defined by equations with coefficients in $\mathbb{Q}$. A polyhedral set is a finite union of polyhedra. A polytope is the convex hull of a finite set of points. A convex polyhedral cone is the convex conic set generated by a finite set of vectors of $N_{\mathbb{R}}$.

The following theorem is a basic tool in the study of polyhedra. 
Theorem 2.1 (Minkowski-Weyl) Let $E$ be a subset of $N_{\mathbb{R}}$. Then $E$ is a polyhedron if and only if there exist a polytope $\Delta$ and a convex polyhedral cone $\sigma$ such that

$$
E=\Delta+\sigma
$$

An immediate consequence of this result is that the notion of polytope coincides with that of bounded polyhedron and the notion of convex polyhedral cone coincides with that of conic polyhedron.

Let $\Lambda$ be a polyhedron. The relative interior of $\Lambda$, denoted $\operatorname{ri}(\Lambda)$, is defined as the interior of $\Lambda$ relative to the minimal affine space that contains it. For $x \in M_{\mathbb{R}}$, we set

$$
\Lambda_{x}=\{u \in \Lambda \mid\langle x, u\rangle \leq\langle x, v\rangle, \forall v \in \Lambda\} .
$$

A nonempty subset $F \subset \Lambda$ is called a face of $\Lambda$ if it is of the form $\Lambda_{x}$ for some $x \in M_{\mathbb{R}}$. The polyhedron $\Lambda$ is the disjoint union of the relative interior of its faces.

Let $E \subset N_{\mathbb{R}}$ be a polyhedral subset. For each $p \in E$, the local recession cone of $E$ at $p$ is defined as

$$
\operatorname{rec}_{p}(E)=\left\{u \in N_{\mathbb{R}} \mid p+\lambda u \in E, \forall \lambda \geq 0\right\} .
$$

The recession cone of $E$ is defined as

$$
\operatorname{rec}(E)=\bigcap_{p \in E} \operatorname{rec}_{p}(E) .
$$

Observe that both the local and the global recession cones are conic subsets of $N_{\mathbb{R}}$. If $\Lambda$ is a polyhedron, we have the alternative characterization

$$
\operatorname{rec}(\Lambda)=\left\{u \in N_{\mathbb{R}} \mid \Lambda+u \subset \Lambda\right\} .
$$

Hence, for polyhedra, the above definition agrees with the notion of recession cone from convex analysis [6]. Moreover, $\operatorname{rec}(\Lambda)$ agrees with the cone $\sigma$ in Theorem 3.4. In particular, $\sigma$ is determined by $\Lambda$, and $\operatorname{rec}(\Lambda)$ is a convex polyhedral cone. A further consequence of the Minkowski-Weyl theorem is that, for polyhedra, local and global recession cones agree. In other words, $\operatorname{rec}_{p}(\Lambda)=\operatorname{rec}(\Lambda)$ for any $p \in \Lambda$. Observe that, if $\Lambda$ is rational or strongly convex, the same is true for $\operatorname{rec}(\Lambda)$.

To a polyhedral subset $E \subset N_{\mathbb{R}}$ we can also associate the (not necessarily convex) polyhedral cone of $N_{\mathbb{R}} \times \mathbb{R}$ given by

$$
\mathrm{c}(E)=\overline{\mathbb{R}_{>0}(E \times\{1\})} \subset N_{\mathbb{R}} \times \mathbb{R}_{\geq 0} .
$$

It is called the cone of $E$. If $\Lambda$ is a polyhedron, then $\operatorname{rec}(\Lambda)=\mathrm{c}(\Lambda) \cap\left(N_{\mathbb{R}} \times\{0\}\right)$. This is not true for general polyhedral sets. Again, if $\Lambda$ is rational or strongly convex, the same is true for $\mathrm{c}(\Lambda)$.

Definition 2.2 A polyhedral complex in $N_{\mathbb{R}}$ is a nonempty collection $\Pi$ of polyhedra of $N_{\mathbb{R}}$ such that

(1) every face of an element of $\Pi$ is also in $\Pi$, 
(2) any two elements of $\Pi$ are either disjoint or intersect in a common face.

A polyhedral complex $\Pi$ is called rational (respectively strongly convex, conic) if all of its elements are rational (respectively strongly convex, cones). A strongly convex conic polyhedral complex is called a fan. For shorthand, strongly convex rational will be abbreviated to SCR.

The support of $\Pi$ is the polyhedral set

$$
|\Pi|=\bigcup_{\Lambda \in \Pi} \Lambda
$$

For a subset $E \subset N_{\mathbb{R}}$, we say that $\Pi$ is a polyhedral complex in $E$ whenever $|\Pi| \subset E$. We say that a complex in $E$ is complete if $|\Pi|=E$.

\section{Complexes and Fans}

There are two natural processes for linearizing a polyhedral complex. Intuitively, the first one is to look at the complex from far away, so that the unbounded polyhedra became cones. In precise terms, the recession of $\Pi$ is defined as the collection of polyhedral cones of $N_{\mathbb{R}}$

$$
\operatorname{rec}(\Pi)=\{\operatorname{rec}(\Lambda) \mid \Lambda \in \Pi\}
$$

The second process is analogous to the linearization of an affine space. The cone of $\Pi$ is defined as the collection of cones in $N_{\mathbb{R}} \times \mathbb{R}$

$$
\mathrm{c}(\Pi)=\{\mathrm{c}(\Lambda) \mid \Lambda \in \Pi\} \cup\{\sigma \times\{0\} \mid \sigma \in \operatorname{rec}(\Pi)\} .
$$

It is a natural question to ask whether the recession or the cone of a given polyhedral complex is a complex too. The following example shows that this is not always the case.

Example 3.1 Let $\Pi$ be the polyhedral complex in $\mathbb{R}^{3}$ consisting in the set of faces of the polyhedra

$$
\Lambda_{1}:=\left\{\left(x_{1}, x_{2}, 0\right) \mid x_{1}, x_{2} \geq 0\right\}, \quad \Lambda_{2}:=\left\{\left(x_{1}, x_{2}, 1\right) \mid x_{1}+x_{2}, x_{1}-x_{2} \geq 0\right\} .
$$

We have that $\operatorname{rec}\left(\Lambda_{1}\right)$ and $\operatorname{rec}\left(\Lambda_{2}\right)$ are two cones in $\mathbb{R}^{2} \times\{0\}$ whose intersection is the cone $\left\{\left(x_{1}, x_{2}, 0\right) \mid x_{2}, x_{1}-x_{2} \geq 0\right\}$. This cone is neither a face of $\operatorname{rec}\left(\Lambda_{1}\right)$ nor of $\operatorname{rec}\left(\Lambda_{2}\right)$. Hence $\operatorname{rec}(\Pi)$ is not a complex, and consequently, neither is $\mathrm{c}(\Pi)$. In Fig. 1 we see the polyhedron $\Lambda_{1}$ in light grey, the polyhedron $\Lambda_{2}$ in darker grey, and $\operatorname{rec}\left(\Lambda_{2}\right)$ as dashed lines.

This example shows that we need to impose some condition on $\Pi$ if we want to ensure that $\operatorname{rec}(\Pi)$ and $\mathrm{c}(\Pi)$ are complexes. The precise hypothesis in Theorem 3.4 was suggested to us by Francisco Santos. The key observation is that it is enough to assume that $|\Pi|$ satisfies a version of the Minkowski-Weyl theorem. 
Fig. 1 A polyhedral complex whose recession is not a fan

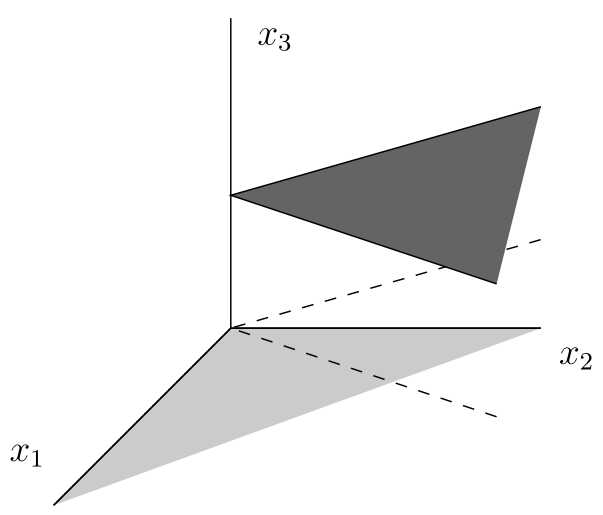

Lemma 3.2 Let $E$ be a polyhedral set. The following conditions are equivalent.

(1) There is a decomposition $E=\Delta+\sigma$, where $\Delta$ is a finite union of polytopes, and $\sigma$ is a convex polyhedral cone.

(2) $\operatorname{rec}_{p}(E)=\operatorname{rec}(E)$ for all $p \in E$.

Proof We first prove that (1) implies (2). Let $E=\Delta+\sigma$ be as in (1). Since $\sigma$ is a convex cone, it is a semigroup. This implies that $\sigma \subset \operatorname{rec}_{p}(E)$ for all $p \in E$. Since $\Delta$ is compact and $\sigma$ is closed, if $v \in \operatorname{rec}_{p}(E)$, the fact that the ray $p+\mathbb{R}_{\geq 0} v$ is contained in $\Delta+\sigma$ implies that $v \in \sigma$. Hence $\operatorname{rec}_{p}(E)=\sigma$ for all $p$, and $\operatorname{so} \operatorname{rec}(E)=\sigma=$ $\operatorname{rec}_{p}(E)$.

Conversely, write $E=\bigcup_{i} \Lambda_{i}$. We set $\sigma=\operatorname{rec}(E)=\operatorname{rec}_{p}(E)$ for any $p \in E$ and $\sigma_{i}=\operatorname{rec}\left(\Lambda_{i}\right)$ for each $i$. We have that $\sigma_{i} \subset \sigma$ for all $i$. By the Minkowski-Weyl theorem, for each $i$, there exists a polytope $\Delta_{i} \subset \Lambda_{i}$ such that $\Lambda_{i}=\Delta_{i}+\sigma_{i}$. Consider the finite union of polytopes $\Delta=\bigcup_{i} \Delta_{i}$. It is clear that $E \subset \Delta+\sigma$. Besides, $\Delta+$ $\sigma \subset E$ since $\Delta$ is contained in $E$. Hence $E=\Delta+\sigma$, as stated.

Definition 3.3 A polyhedral subset $E$ of $N_{\mathbb{R}}$ satisfies the Minkowski-Weyl condition if it satisfies any of the equivalent conditions in Lemma 3.2.

Theorem 3.4 Let $\Pi$ be a polyhedral complex in $N_{\mathbb{R}}$ such that $|\Pi|$ is a connected polyhedral set satisfying the Minkowski-Weyl condition. Then

(1) $\operatorname{rec}(\Pi)$ is a conic polyhedral complex in $N_{\mathbb{R}}$, and $|\operatorname{rec}(\Pi)|=\operatorname{rec}(|\Pi|)$.

(2) $\mathrm{c}(\Pi)$ is a conic polyhedral complex in $N_{\mathbb{R}} \times \mathbb{R}_{\geq 0}$, and $|\mathrm{c}(\Pi)|=\mathrm{c}(|\Pi|)$.

(3) If, in addition, $\Pi$ is rational (respectively strongly convex), then both $\operatorname{rec}(\Pi)$ and $\mathrm{c}(\Pi)$ are rational (respectively fans).

We need some lemmas before starting the proof of this result.

Lemma 3.5 Let $\Lambda \subset N_{\mathbb{R}}$ be a polyhedron. Then the collection of the recession cones of the form $\operatorname{rec}(F)$ for some face $F$ of $\Lambda$ coincides with the set of faces of $\operatorname{rec}(\Lambda)$. In particular, it is a complex. 
Proof By the Minkowski-Weyl theorem, there exist a polytope $\Delta$ and a cone $\sigma$ such that $\Lambda=\Delta+\sigma$. It is easy to verify that, if $x \in M_{\mathbb{R}}$, then $\Lambda_{x}=\Delta_{x}+\sigma_{x}$. Therefore, $\operatorname{rec}\left(\Lambda_{x}\right)=\sigma_{x}$, which implies the result.

Lemma 3.6 Let $\Lambda$ be a polyhedron, and $v \in \operatorname{rec}(\Lambda)$. Then there is a face $F$ of $\Lambda$ such that $v \in \operatorname{ri}(\operatorname{rec}(F))$. Moreover, if $F_{1}$ and $F_{2}$ are two faces satisfying this condition, then $\operatorname{rec}\left(F_{1}\right)=\operatorname{rec}\left(F_{2}\right)$.

Proof This easily follows from Lemma 3.5.

Lemma 3.7 [6, Theorem 18.1] Let $C$ be a convex set, and $F$ a face of $C$. If $D \subset C$ is a convex subset such that $\operatorname{ri}(D) \cap F \neq \emptyset$, then $D \subset F$.

Proof of Theorem 3.4 We first prove (1). The sets $\operatorname{rec}(\Lambda)$ for $\Lambda \in \Pi$ are polyhedra, and, by Lemma 3.5, any face of an element of $\operatorname{rec}(\Pi)$ is in $\operatorname{rec}(\Pi)$. Thus, $\operatorname{rec}(\Pi)$ satisfies the first condition in Definition 2.2, and we have to show that it also satisfies the second one. That is, we have to show that given $\Lambda_{1}, \Lambda_{2} \in \Pi \operatorname{such}$ that $\operatorname{rec}\left(\Lambda_{1}\right) \cap$ $\operatorname{rec}\left(\Lambda_{2}\right)$ is nonempty, this intersection is a common face of $\operatorname{rec}\left(\Lambda_{1}\right)$ and $\operatorname{rec}\left(\Lambda_{2}\right)$.

Let $v \in \operatorname{ri}\left(\operatorname{rec}\left(\Lambda_{1}\right) \cap \operatorname{rec}\left(\Lambda_{2}\right)\right)$. Let $F_{i}, i=1,2$, be faces of $\Lambda_{i}$ satisfying the condition of Lemma 3.6. We claim that we can find a finite sequence of polyhedra $\Gamma_{1}, \ldots, \Gamma_{n+1}$ with the following properties:

(1) $F_{1}$ is a face of $\Gamma_{1}$, and $F_{2}$ is a face of $\Gamma_{n+1}$.

(2) The vector $v$ belongs to $\operatorname{rec}\left(\Gamma_{i} \cap \Gamma_{i+1}\right)$ for $i=1, \ldots, n$.

To prove this claim, we choose points $p_{i} \in \operatorname{ri}\left(F_{i}\right), i=1,2$. Since $|\Pi|$ is a connected polyhedral set, we can find a polygonal path $\gamma:[0,1] \rightarrow|\Pi|$ joining $p_{1}$ and $p_{2}$. By construction, $v \in \operatorname{rec}_{p_{1}}(|\Pi|)$. Since $|\Pi|$ satisfies the MinkowskiWeyl condition, $v \in \operatorname{rec}(|\Pi|)$. Therefore the map $S:[0,1] \times \mathbb{R}_{\geq 0} \rightarrow|\Pi|$, given by $S(t, r)=\gamma(t)+r v$, is well defined. This is the key step in this proof, and it fails if the hypothesis are not fulfilled: if $|\Pi|$ is not connected, then the polygonal path $\gamma$ may not exist, and if $|\Pi|$ is connected but does not satisfy the Minkowski-Weyl condition, then the map $S$ may not exist.

Since $S$ is a piecewise affine function, we can find a finite covering $\mathfrak{U}$ of $[0,1] \times$ $\mathbb{R}_{\geq 0}$ by polyhedra such that, for each $K \in \mathfrak{U}$, there is a $\Lambda \in \Pi$ with $S(K) \subset \Lambda$. By the finiteness of $\mathfrak{U}$, we can find a number $l \geq 0$ such that the restriction of the covering $\mathfrak{U}$ to $[0,1] \times[l, \infty)$ consists of sets of the form $I_{\alpha} \times[l, \infty)$, where the $I_{\alpha}$ are closed intervals that cover $[0,1]$. We choose $I_{1}, \ldots, I_{n+1}$ among them such that $0 \in I_{0}, 1 \in I_{n+1}$, and $I_{i} \cap I_{i+1} \neq 0$ for $i=1, \ldots, n$. For each $i$, we choose a polyhedron $\Gamma_{i} \in \Pi$ such that $S\left(I_{i} \times[l, \infty)\right)$ is contained in $\Gamma_{i}$.

Since $\Gamma_{0}$ contains the point $p_{1}+l v$ and this point belongs to $\operatorname{ri}\left(F_{1}\right)$, we have that $F_{1}$ is a face of $\Gamma_{0}$. Analogously, $F_{2}$ is a face of $\Gamma_{n+1}$. By construction, it is also clear that $v \in \operatorname{rec}\left(\Gamma_{i} \cap \Gamma_{i+1}\right)$. Thus the claim is proved.

For each $i=1, \ldots, n$, we choose a face $G_{i}$ of $\Gamma_{i} \cap \Gamma_{i+1}$ that satisfies the condition of Lemma 3.6. Applying Lemma 3.6 to the polyhedra $\Gamma_{i}$, we obtain

$$
\operatorname{rec}\left(F_{1}\right)=\operatorname{rec}\left(G_{1}\right)=\cdots=\operatorname{rec}\left(G_{n}\right)=\operatorname{rec}\left(F_{2}\right) .
$$


By Lemma 3.7, $\operatorname{rec}\left(\Lambda_{1}\right) \cap \operatorname{rec}\left(\Lambda_{2}\right) \subset \operatorname{rec}\left(F_{1}\right)$. Thus, we have the chain of inclusions

$$
\operatorname{rec}\left(\Lambda_{1}\right) \cap \operatorname{rec}\left(\Lambda_{2}\right) \subset \operatorname{rec}\left(F_{1}\right) \cap \operatorname{rec}\left(F_{2}\right) \subset \operatorname{rec}\left(\Lambda_{1}\right) \cap \operatorname{rec}\left(\Lambda_{2}\right) .
$$

Hence $\operatorname{rec}\left(\Lambda_{1}\right) \cap \operatorname{rec}\left(\Lambda_{2}\right)=\operatorname{rec}\left(F_{1}\right)=\operatorname{rec}\left(F_{2}\right)$ is a common face of $\operatorname{rec}\left(\Lambda_{1}\right)$ and $\operatorname{rec}\left(\Lambda_{2}\right)$. We conclude that $\operatorname{rec}(\Pi)$ is a complex.

We now prove that $|\operatorname{rec}(\Pi)|=\operatorname{rec}(|\Pi|)$. On the one hand, for any $p \in|\Pi|$, we always have the chain of inclusions

$$
\operatorname{rec}(|\Pi|) \subset \operatorname{rec}_{p}(|\Pi|) \subset \bigcup_{\Lambda \in \Pi} \operatorname{rec}(\Lambda)=|\operatorname{rec}(\Pi)| .
$$

On the other hand, if $\Lambda \in \Pi$ and $p \in \Lambda$, we have $\operatorname{rec}(\Lambda) \subset \operatorname{rec}_{p}(|\Pi|)=\operatorname{rec}(|\Pi|)$, where the second equality follows from the Weyl-Minkowski condition. Thus

$$
|\operatorname{rec}(\Pi)|=\bigcup_{\Lambda \in \Pi} \operatorname{rec}(\Lambda) \subset \operatorname{rec}(|\Pi|) .
$$

Hence the equality.

We next prove (2). For a polyhedron $\Lambda$, we denote $\mathrm{c}^{\circ}(\Lambda)=\mathbb{R}_{>0}(\Lambda \times\{1\})$. Observe that

$$
\mathrm{c}(\Lambda)=\mathrm{c}^{\circ}(\Lambda) \sqcup(\operatorname{rec}(\Lambda) \times\{0\}) .
$$

By Lemma 3.5, every face of $\operatorname{rec}(\Lambda) \times\{0\}$ is of the form $\operatorname{rec}(F) \times\{0\}$ for some face $F$ of $\Lambda$. Moreover, a face of c $(\Lambda)$ is either of the form c $(F)$ for a face $F$ of $\Lambda$ or a face of $\operatorname{rec}(\Lambda) \times\{0\}$. Hence $c(\Pi)$ satisfies the first condition in Definition 2.2.

It remains to prove that any two not disjoint elements of $\mathrm{c}(\Pi)$ intersect in a common face. By (1), the intersection of two cones of c $(\Pi)$ contained in $N_{\mathbb{R}} \times\{0\}$ is a common face. By (3.8), the same is true if we intersect a cone contained in $N_{\mathbb{R}} \times\{0\}$ with a cone of the form $\mathrm{c}(\Lambda)$. If $\Lambda_{1}, \Lambda_{2} \in \Pi$, one verifies using (3.8) that

$$
\mathrm{c}\left(\Lambda_{1}\right) \cap \mathrm{c}\left(\Lambda_{2}\right)= \begin{cases}\mathrm{c}\left(\Lambda_{1} \cap \Lambda_{2}\right) & \text { if } \Lambda_{1} \cap \Lambda_{2} \neq \varnothing, \\ \left(\operatorname{rec}\left(\Lambda_{1}\right) \cap \operatorname{rec}\left(\Lambda_{2}\right)\right) \times\{0\} & \text { otherwise. }\end{cases}
$$

In both cases, this is a common face of $\mathrm{c}\left(\Lambda_{1}\right)$ and $\mathrm{c}\left(\Lambda_{2}\right)$. Hence $\mathrm{c}(\Pi)$ is a complex.

Statement (3) follows easily from the previous ones.

\section{Example 3.9}

(1) Let $\Pi$ be the complex in $\mathbb{R}^{3}$ consisting of the set of faces of the polyhedra $\left\{\left(x_{1}, x_{2}, 0\right) \mid x_{1}, x_{2} \geq 0\right\},\left\{\left(x_{1}, x_{2}, 1\right) \mid x_{1} \geq x_{2} \geq 0\right\}$ and $\left\{\left(x_{1}, x_{2}, 1\right) \mid x_{2} \geq x_{1} \geq 0\right\}$. This polyhedral complex satisfies the Minkowski-Weyl condition, but $|\Pi|$ is not connected, and $\operatorname{rec}(\Pi)$ is not a complex. Therefore, the connectedness assumption is necessary for the conclusion of Theorem 3.4.

(2) Let $\Pi$ be the complex in $\mathbb{R}^{3}$ consisting of the set of faces of the polyhedra $\left\{\left(x_{1}, x_{2}, 0\right) \mid x_{1} \geq x_{2} \geq 0\right\}$ and $\left\{\left(x_{1}, x_{2}, 1\right) \mid x_{2} \geq x_{1} \geq 0\right\}$. This polyhedral complex does not satisfy the Minkowski-Weyl condition, $\operatorname{rec}(\Pi)$ is a fan, but $\operatorname{rec}(|\Pi|) \varsubsetneqq$ $|\operatorname{rec}(\Pi)|$. 
Any polyhedron satisfies the Minkowski-Weyl condition, and therefore we have:

Corollary 3.10 Let $\Pi$ be a polyhedral complex in $N_{\mathbb{R}}$ such that $|\Pi|$ is convex. Then $\operatorname{rec}(\Pi)$ and $\mathrm{c}(\Pi)$ are conic polyhedral complexes. If, in addition, $\Pi$ is rational (respectively strongly convex), then $\operatorname{rec}(\Pi)$ and $\mathrm{c}(\Pi)$ are rational (respectively fans).

Let $\Sigma$ be a conic polyhedral complex in $N_{\mathbb{R}} \times \mathbb{R}_{\geq 0}$. We denote by aff $(\Sigma)$ the complex in $N_{\mathbb{R}}$ obtained by intersecting $\Sigma$ with the hyperplane $N_{\mathbb{R}} \times\{1\}$. Again, if $\Sigma$ is rational or strongly convex, the same is true for aff $(\Sigma)$.

Corollary 3.11 The correspondence $\Pi \mapsto \mathrm{c}(\Pi)$ is a bijection between the set of complete polyhedral complexes in $N_{\mathbb{R}}$ and the set of complete conic polyhedral complexes in $N_{\mathbb{R}} \times \mathbb{R}_{\geq 0}$. Its inverse is the correspondence aff. These bijections preserve rationality and strong convexity.

Proof By Theorem 3.4, if $\Pi$ is a complete polyhedral complex in $N_{\mathbb{R}}$, then $\mathrm{c}(\Pi)$ is also a complete conic polyhedral complex in $N_{\mathbb{R}} \times \mathbb{R}_{\geq 0}$. Conversely, if $\Sigma$ is a complete conic polyhedral complex in $N_{\mathbb{R}} \times \mathbb{R}_{\geq 0}$, it is clear that aff $(\Sigma)$ is a complete polyhedral complex in $N_{\mathbb{R}}$.

If $\Pi$ is a complete polyhedral complex in $N_{\mathbb{R}}$, then $\Pi=\operatorname{aff}(\mathrm{c}(\Pi))$. It remains to verify that the other composition is the identity. Since we already know that c $(\operatorname{aff}(\Sigma))$ is a complex in $N_{\mathbb{R}} \times \mathbb{R}_{\geq 0}$, it is enough to show that $\Sigma$ and c(aff( $\left.(\Sigma)\right)$ have the same cones of dimension $n+1$. But this is obvious. Hence $\Sigma=\mathrm{c}(\operatorname{aff}(\Sigma))$.

The last statement is clear.

\section{Remark 3.12}

(1) Example 3.1 shows that the map $\Pi \mapsto \mathrm{c}(\Pi)$ does not produce a conic polyhedral complex from an arbitrary polyhedral complex. Thus the above corollary cannot be extended to arbitrary complexes.

(2) Given a conic polyhedral complex $\Sigma$, the polyhedral complex aff $(\Sigma)$ does not need to satisfy the hypotheses of Theorem 3.4. Nevertheless, $\mathrm{c}(\operatorname{aff}(\Sigma))$ is the conic complex $\Sigma$. Therefore the hypotheses of Theorem 3.4 are sufficient for the recession being a complex, but they are not necessary. It would be interesting to have a full characterization of the complexes that arise as the image of aff.

(3) If we restrict to polyhedral complexes with convex support, the correspondence aff is not injective, and $\mathrm{c}$ is only a right inverse of aff. This is why in Corollary 3.11, we restrict ourselves to complete complexes.

Let $\Pi$ be a polyhedral complex. As we have seen in Remark 3.12 (2), the hypotheses of Theorem 3.4 are not necessary for $\operatorname{rec}(\Pi)$ being a complex. A class of examples for which this is true is that of extendable complexes.

Definition 3.13 A polyhedral complex $\Pi$ in $\mathbb{R}^{n}$ is called extendable if there exists a complete polyhedral complex $\bar{\Pi}$ such that $\Pi$ is a subcomplex of $\bar{\Pi}$.

Corollary 3.14 Let $\Pi$ be an extendable polyhedral complex. Then both $\operatorname{rec}(\Pi)$ and $\mathrm{c}(\Pi)$ are complexes. 
Proof If $\sigma \in \operatorname{rec}(\Pi)$, then it is clear that all the faces of $\sigma$ also belong to $\operatorname{rec}(\Pi)$. Let $\bar{\Pi}$ be a complete polyhedral complex which contains $\Pi$. Let $\sigma, \tau \in \operatorname{rec}(\Pi)$. Since both belong to $\operatorname{rec}(\bar{\Pi})$, by Theorem 3.4 , its intersection is a common face. Thus $\operatorname{rec}(\Pi)$ is a complex, and the same is true for $\mathrm{c}(\Pi)$.

In particular, the complex from Example 3.1 cannot be extended to a complete polyhedral complex. By contrast, the complex from Example 3.9 (2) is extendable. This last example shows that an extendable complex $\Pi$ does not necessarily satisfy that $|\operatorname{rec}(\Pi)|=\operatorname{rec}(|\Pi|)$.

If one is willing to admit subdivisions, the issues raised by Example 3.1 disappear.

Proposition 3.15 Let $\Pi$ be a polyhedral complex in $\mathbb{R}^{n}$. Then there exists a subdivision $\Pi^{\prime}$ of $\Pi$ that is extendable. In particular, $\operatorname{rec}\left(\Pi^{\prime}\right)$ and $\mathrm{c}\left(\Pi^{\prime}\right)$ are complexes.

Proof Let $\Lambda_{1}, \ldots, \Lambda_{m}$ be the polyhedra of $\Pi$. For each $i$, the complex defined by the faces of $\Lambda_{i}$ is extendable. Denote by $\Pi_{i}$ any such extension. Then

$$
\bar{\Pi}:=\left\{\Gamma_{1} \cap \cdots \cap \Gamma_{m} \mid \Gamma_{i} \in \Pi_{i}\right\}
$$

is a complete polyhedral complex that is a common subdivision of the $\Pi_{i}$. Let $\Pi^{\prime}$ be the set of polyhedra of $\bar{\Pi}$ that are contained in $|\Pi|$. Then $\Pi^{\prime}$ is an extendable subdivision of $\Pi$. The last statement follows from Corollary 3.14.

\section{Toric Schemes over a DVR and Tropical Varieties}

Let $K$ be a field provided with a nontrivial discrete valuation val: $K^{\times} \rightarrow \mathbb{Z}$. Let $K^{\circ}$ be its valuation ring, and $S=\operatorname{Spec}\left(K^{\circ}\right)$ its base scheme. Let $\mathbb{T}_{S} \simeq \mathbb{G}_{m, S}^{n}$ be a split torus over $S$, and let $\mathbb{T}=\mathbb{T}_{S} \times \operatorname{Spec}(K)$ be the corresponding split torus over $K$. Let $N=\operatorname{Hom}\left(\mathbb{G}_{m, K}, \mathbb{T}\right)$ be the lattice of one-parameter subgroups of $\mathbb{T}$.

Definition 4.1 A toric scheme over $S$ of relative dimension $n$ is a normal integral separated $S$-scheme of finite type $\mathcal{X}$ equipped with an open embedding $\mathbb{T} \hookrightarrow \mathcal{X} \times$ $\operatorname{Spec}(K)$ and an $S$-action of $\mathbb{T}_{S}$ over $\mathcal{X}$ that extends the action of $\mathbb{T}$ on itself.

Toric schemes over a DVR were introduced and studied in [3]. In loc. cit., to each rational fan $\Sigma$ in $N_{\mathbb{R}} \times \mathbb{R}_{\geq 0}$ it is associated a toric scheme $\mathcal{X}_{\Sigma}$ over $S$. Moreover, the following classification theorem is proved.

Theorem 4.2 ([3, § IV.3]) The correspondence $\Sigma \mapsto \mathcal{X}_{\Sigma}$ is a bijection between the set of rational fans in $N_{\mathbb{R}} \times \mathbb{R}_{\geq 0}$ and the set of toric schemes over $S$ of relative dimension $n$. The scheme $\mathcal{X}_{\Sigma}$ is proper if and only if $\Sigma$ is complete.

We are interested in the question of when an SCR polyhedral complex in $N_{\mathbb{R}}$ defines a toric scheme over $S$ and whether this assignment allows one to classify toric schemes over $S$. Example 3.1 shows that an SCR polyhedral complex of $N_{\mathbb{R}}$ does not necessarily define a toric scheme over $S$. Theorem 3.4 shows that to each SCR 
polyhedral complex $\Pi$ in $N_{\mathbb{R}}$ such that $|\Pi|$ is connected and satisfies the MinkowskiWeyl condition we can associate a toric scheme $\mathcal{X}_{\mathrm{c}(\Pi)}$. Remark 3.12 shows that this assignment cannot give a classification in full generality. A direct consequence of Theorem 4.2 and Corollary 3.11 is the following classification result.

Theorem 4.3 The correspondence $\Pi \mapsto \mathcal{X}_{\mathrm{c}(\Pi)}$ is a bijection between the set of complete $S C R$ polyhedral complexes of $N_{\mathbb{R}}$ and the set of proper toric schemes over $S$ of relative dimension $n$.

Polyhedral complexes play also an important role in tropical geometry. Observe that the valuation of $K$ induces a map $\mathbb{T}(\bar{K}) \rightarrow N_{\mathbb{R}}$, which we also denote by val.

Definition 4.4 Let $V \subset \mathbb{T}$ be an algebraic set. The tropical variety associated to $V$, denoted trop $(V)$, is the closure in $N_{\mathbb{R}}$ of the subset val $(V(\bar{K}))$.

Tropical varieties are polyhedral sets that can be equipped with a standard structure of a polyhedral complex. Let $G_{V}$ denote the so-called Gröbner complex of $V$ [4, § 2], [2, Definition 5.5]. This is a complete rational polyhedral complex in $N_{\mathbb{R}}$. If the stabilizer of $V$ is trivial, then $G_{V}$ is strongly convex. The tropical variety trop $(V)$ is the union of a finite number of elements of $G_{V}$, and so it inherits a structure of a polyhedral complex. We denote by $\Pi_{V}$ this extendable polyhedral complex. As a consequence of Theorem 3.4 and Corollary 3.14, we deduce the following:

Proposition 4.5 Let $V \subset \mathbb{T}$ be an algebraic set, then $\mathrm{c}\left(\Pi_{V}\right)$ is a rational conic polyhedral complex. Moreover, let $G_{V}^{\prime}$ be an SCR subdivision of $G_{V}$, and let $\Pi_{V}^{\prime}$ be the subdivision of $\Pi_{V}$ induced by $G_{V}^{\prime}$. Then $\mathrm{c}\left(\Pi_{V}^{\prime}\right)$ is a rational fan. In particular, if the stabilizer of $V$ is trivial, then $\mathrm{c}\left(\Pi_{V}\right)$ is a rational fan.

Hence, if the stabilizer of $V$ is trivial, we can associate to $V$ the toric scheme $\mathcal{X}_{\mathrm{c}\left(\Pi_{V}\right)}$. This kind of schemes are interesting because, by a theorem of J. Tevelev extended by D. Speyer, the closure $\mathcal{V}$ of $V$ in $\mathcal{X}$ is proper over $S$ [7, Proposition 2.4.1], [1, Proposition 3.2]. The scheme $\mathcal{V}$ is called a tropical compactification of $V$.

Acknowledgements We thank Eric Katz and Francisco Santos for enlightening discussions. We also thank an anonymous referee for useful suggestions. This research was done during a stay of the first author at the University of Barcelona. We thank this institution for its hospitality.

\section{References}

1. Helm, D., Katz, E.: Monodromy filtrations and the topology of tropical varieties. Can. J. Math., to appear. arXiv:0804.3651v4

2. Katz, E.: A tropical toolkit. Expo. Math. 27, 1-36 (2009)

3. Kempf, G., Knudsen, F.F., Mumford, D., Saint-Donat, B.: Toroidal Embeddings. I. Lecture Notes in Mathematics, vol. 339. Springer, Berlin (1973)

4. Maclagan, D., Sturmfels, B.: Introduction to Tropical Geometry. Draft (2009)

5. Nishinou, T., Siebert, B.: Toric degenerations of toric varieties and tropical curves. Duke Math. J. 135, 1-51 (2006)

6. Rockafellar, R.T.: Convex Analysis. Princeton University Press, Princeton (1970)

7. Speyer, D.: Tropical geometry. Ph.D. thesis, UC Berkeley (2005) 